УДК 517.91

\title{
ЗАДАЧА НЕЙМАНА ДЛЯ ОБЫКНОВЕННОГО ДИФФЕРЕНЦИАЛЬНОГО УРАВНЕНИЯ ДРОБНОГО ПОРЯДКА
}

\section{Л. Х. Гадзова}

Решена задача Неймана для обыкновенного дифференциального уравнения дробного порядка с постоянными коэффициентами. Построена функция Грина, доказана конечность числа вещественных собственных значений.

Ключевые слова: краевая задача, оператор дробного дифференцирования, оператор Римана Лиувилля, оператор Капуто.

1. Постановка задачи. В интервале $0<x<1$ рассмотрим уравнение

$$
\sum_{j=1}^{m} \beta_{j} \partial_{0 x}^{\alpha_{j}} u(x)+\lambda u(x)=f(x),
$$

где $\left.\alpha_{j} \in\right] 1,2\left[, \lambda, \beta_{j} \in \mathbb{R}, \beta_{1}>0, \alpha_{1}>\alpha_{2}>\cdots>\alpha_{m}, \partial_{0 x}^{\gamma} u(x)\right.$ - регуляризованная дробная производная (производная Капуто) [1, с. 11]:

$$
\partial_{0 x}^{\gamma} u(x)=D_{0 x}^{\gamma-n} u^{(n)}(x), \quad n-1<\gamma \leqslant n,
$$

где $D_{0 x}^{\gamma}$ - оператор дробного интегро-дифференцирования порядка $\gamma$ в смысле Римана Лиувилля $[1$, с. 9] по переменной $x$, определяемый равенством:

$$
D_{0 x}^{\gamma} u(x)= \begin{cases}\frac{1}{\Gamma(-\gamma)} \int_{0}^{x} u(t)(x-t)^{-\gamma-1} d t, & \gamma<0, \\ u(x), & \gamma=0, \\ \frac{d^{n}}{d x^{n}} D_{0 x}^{\gamma-n} u(t), & n-1<\gamma \leqslant n, n \in \mathbb{N} .\end{cases}
$$

Работа [2] играет важную роль в исследованиях, посвященных дифференциальным уравнениям дробного порядка. В работах $[3,4]$ были изучены линейные дифференциальные уравнения дробного порядка. Краевая задача для обыкновенного дифференциального уравнения второго порядка, содержащего группу младших членов с производными Римана - Лиувилля, исследовалась в работе [5]. В монографии [1, с. 120] даны постановки видоизмененных задач Коши и Неймана для уравнения второго порядка с дробной производной порядка $\alpha \in] 1,2[$. В работах [6-8] исследовались линейные обыкновенные дифференциальные уравнения дробного порядка с постоянными коэффициентами. Относительно изложения результатов и библиографии работ, связанных с линейными дифференциальными уравнениями дробного порядка, укажем работы [9] и [10].

\footnotetext{
(c) 2016 Гадзова Л. Х.

${ }^{1}$ Работа выполнена при финансовой поддержке Российского фонда фундаментальных исследований, проект № 16-01-00462.
} 
Для уравнения (1) решена задача Дирихле методом функции Грина в работе [11], также найдено условие однозначной разрешимости [12].

Интерес к исследованию уравнений дробного порядка стимулируется практическими приложениями, которые обнаруживаются в физике и математическом моделировании фрактальных сред [1, с. 149], [13-15].

В данной работе доказана теорема существования и единственности решения задачи Неймана, получено явное представление решения исследуемой задачи и построена соответствующая функция Грина. Доказано, что исследуемая задача разрешима при любом параметре $\lambda \in \mathbb{R}$, за исключением, быть может, конечного числа значений параметра.

Регулярным решением уравнения (1) назовем функцию $u=u(x)$, имеющую абсолютно непрерывную производную первого порядка на отрезке $[0,1]$ и удовлетворяющую уравнению (1) для всех $x \in] 0,1[$.

ЗАдАчА. Найти регулярное решение уравнения (1) в интервале ]0,1[, удовлетворяющее условиям

$$
u^{\prime}(0)=a, \quad u^{\prime}(1)=b,
$$

где $a, b$ - заданные постоянные.

2. Основной результат. Рассмотрим функцию [12]

$$
\mathscr{G}(x, t)=\frac{1}{\beta_{1}}\left(H(x-t) G_{m}^{\alpha_{1}}(x-t)-G_{m}^{\alpha_{1}-1}(1-t) \frac{1+\nu_{1} G_{m}^{\alpha_{1}+1}(x)}{\nu_{1} G_{m}^{\alpha_{1}}(1)}\right),
$$

где [8]

$$
\begin{gathered}
G_{m}^{\mu}(x)=G_{m}^{\mu}\left(x ; \nu_{1}, \ldots, \nu_{m} ; \gamma_{1}, \ldots, \gamma_{m}\right) \equiv \int_{0}^{\infty} e^{-t} S_{m}^{\mu}\left(x ; \nu_{1} t, \ldots, \nu_{m} t ; \gamma_{1}, \ldots, \gamma_{m}\right) d t, \\
\nu_{1}=-\frac{\lambda}{\beta_{1}}, \quad \nu_{j}=-\frac{\beta_{j}}{\beta_{1}}, \quad \gamma_{1}=\alpha_{1}, \quad \gamma_{j}=\alpha_{1}-\alpha_{j} \quad(j=2, \ldots, m), \\
S_{m}^{\mu}\left(x ; z_{1}, \ldots, z_{m} ; \gamma_{1}, \ldots, \gamma_{m}\right)=\left(h_{1} * h_{2} * \cdots * h_{m}\right)(x), \\
(g * h)(x)=\int_{0}^{x} g(x-t) h(t) d t-\text { свертка Лапласа функций } g(x) \text { и } h(x), \\
\phi(a, b ; z)=\sum_{k=0}^{\infty} \frac{z^{k}}{k ! \Gamma(a k+b)}-\text { функция Райта }[16] ; \\
x>0, \quad z_{i} \in \mathbb{R}, \quad \gamma_{j}>0, \quad \mu_{j}>0, \quad \mu=\sum_{j=1}^{m} \mu_{j}, \\
H(x-t)= \begin{cases}1, & x \geqslant t, \\
0, & x<t\end{cases}
\end{gathered}
$$

Для функции $G_{m}^{\mu}(x)$ справедливы равенства [7, 8]:

$$
G_{m}^{\mu}(x)=O\left(x^{\mu-1}\right) \quad \text { при } \quad x \rightarrow 0,
$$




$$
\begin{gathered}
D_{0 x}^{\nu} G_{m}^{\mu}(x)=G_{m}^{\mu-\nu}(x), \quad \text { если } \quad \mu>\nu, \\
G_{m}^{\mu}(x)-\sum_{i=1}^{m} \nu_{i} D_{0 x}^{-\gamma_{i}} G_{m}^{\mu}(x)=\frac{x^{\mu-1}}{\Gamma(\mu)} .
\end{gathered}
$$

В частности, из (11) следует формула

$$
\sum_{j=1}^{m} \beta_{j} G_{m}^{\mu-\alpha_{j}}(x)+\lambda G_{m}^{\mu}(x)=\frac{\beta_{1} x^{\mu-\alpha_{1}-1}}{\Gamma\left(\mu-\alpha_{1}\right)} .
$$

Теорема 1. 1) Пусть $f(x) \in C] 0,1]$ представима в виде

$$
f(x)=D_{0 x}^{\alpha_{1}-2} g(x), \quad g(x) \in L[0,1],
$$

и выполнено условие

$$
\nu_{1} G_{m}^{\alpha_{1}}(1) \neq 0 .
$$

Тогда существует регулярное решение задачи (1), (3), которое имеет вид

$$
u(x)=\int_{0}^{1} \mathscr{G}(x, t) f(t) d t+a\left[\sum_{j=1}^{m} \beta_{j} D_{1 t}^{\alpha_{j}-2} \mathscr{G}(x, t)\right]_{t=0}-b\left[\sum_{j=1}^{m} \beta_{j} D_{1 t}^{\alpha_{j}-2} \mathscr{G}(x, t)\right]_{t=1} .
$$

2) Решение задачи (1), (3) единственно тогда и только тогда, когда выполняется условие (13).

$\triangleleft$ Для доказательства теоремы покажем справедливость следующих свойств функции $\mathscr{G}(x, t)$.

1. $D_{1 x}^{\alpha_{1}-2} \mathscr{G}(x, t) \in C(\bar{\Omega}), \Omega=\{(x, t): 0<x<1,0<t<1\}$.

Справедливость этого свойства следует из представления (4) и формул (9) и (10).

2. $\mathscr{G}(x, t)$ удовлетворяет соотношению

$$
\lim _{\delta \rightarrow 0}\left[\left.\sum_{j=1}^{m} \beta_{j} D_{1 t}^{\alpha_{j}-1} \mathscr{G}(x, t)\right|_{t=x+\delta}-\left.\sum_{j=1}^{m} \beta_{j} D_{1 t}^{\alpha_{j}-1} \mathscr{G}(x, t)\right|_{t=x-\delta}\right]=1 .
$$

Действительно, в силу (4) и, принимая во внимание (9)-(11), получим

$$
\begin{gathered}
-\lim _{\delta \rightarrow 0} \sum_{j=1}^{m} \frac{\beta_{j}}{\beta_{1}} G_{m}^{\alpha_{1}-\alpha_{j}}(1-x+\delta) \frac{1+\nu_{1} G_{m}^{\alpha_{1}+1}(x)}{\nu_{1} G_{m}^{\alpha_{1}}(1)}-\lim _{\delta \rightarrow 0} \sum_{j=1}^{m} \frac{\beta_{j}}{\beta_{1}} G_{m}^{\alpha_{1}-\alpha_{j}+1}(\delta) \\
+\lim _{\delta \rightarrow 0} \sum_{j=1}^{m} \frac{\beta_{j}}{\beta_{1}} G_{m}^{\alpha_{1}-\alpha_{j}}(1-x-\delta) \frac{1+\nu_{1} G_{m}^{\alpha_{1}+1}(x)}{\nu_{1} G_{m}^{\alpha_{1}}(1)}=\lim _{\delta \rightarrow 0} \sum_{j=1}^{m} \nu_{j} G_{m}^{\alpha_{1}-\alpha_{j}+1}(\delta)=1 .
\end{gathered}
$$

3. При фиксированном значении $x$, функция $\mathscr{G}(x, t)$ является решением уравнения

$$
\sum_{j=1}^{m} \beta_{j} D_{1 t}^{\alpha_{j}} \mathscr{G}(x, t)+\lambda \mathscr{G}(x, t)=0
$$

в интервалах $(0, x)$ и $(x, 1)$. 
С учетом (9)-(12) получаем

$$
\begin{gathered}
\sum_{j=1}^{m} \frac{\beta_{j}}{\beta_{1}} D_{1 t}^{\alpha_{j}}\left[H(x-t) G_{m}^{\alpha_{1}}(x-t)-G_{m}^{\alpha_{1}-1}(1-t) \frac{1+\nu_{1} G_{m}^{\alpha_{1}+1}(x)}{\nu_{1} G_{m}^{\alpha_{1}}(1)}\right] \\
+\frac{\lambda}{\beta_{1}}\left[H(x-t) G_{m}^{\alpha_{1}}(x-t)-G_{m}^{\alpha_{1}-1}(1-t) \frac{1+\nu_{1} G_{m}^{\alpha_{1}+1}(x)}{\nu_{1} G_{m}^{\alpha_{1}}(1)}\right] \\
=H(x-t)\left(\sum_{j=1}^{m} \frac{\beta_{j}}{\beta_{1}} G_{m}^{\alpha_{1}-\alpha_{j}}(x-t)+\frac{\lambda}{\beta_{1}} G_{m}^{\alpha_{1}}(x-t)\right) \\
-\frac{1+\nu_{1} G_{m}^{\alpha_{1}+1}(x)}{\nu_{1} G_{m}^{\alpha_{1}}(1)}\left(\sum_{j=1}^{m} \frac{\beta_{j}}{\beta_{1}} G_{m}^{\alpha_{1}-\alpha_{j}-1}(1-t)+\frac{\lambda}{\beta_{1}} G_{m}^{\alpha_{1}-1}(1-t)\right)=0 .
\end{gathered}
$$

4. $\mathscr{G}(x, t)$ удовлетворяет краевым условиям

$$
\left[\sum_{j=1}^{m} \beta_{j} D_{1 t}^{\alpha_{j}-1} \mathscr{G}(x, t)\right]_{t=1}=0, \quad\left[\sum_{j=1}^{m} \beta_{j} D_{1 t}^{\alpha_{j}-1} \mathscr{G}(x, t)\right]_{t=0}=0 .
$$

Действительно, учитывая формулы (9) и (12), имеем

$$
\begin{gathered}
{\left[\sum_{j=1}^{m} \frac{\beta_{j}}{\beta_{1}}\left(H(x-t) G_{m}^{\alpha_{1}-\alpha_{j}+1}(x-t)-G_{m}^{\alpha_{1}-\alpha_{j}}(1-t) \frac{1+\nu_{1} G_{m}^{\alpha_{1}+1}(x)}{\nu_{1} G_{m}^{\alpha_{1}}(1)}\right)\right]_{t=1}} \\
=-\frac{1+\nu_{1} G_{m}^{\alpha_{1}+1}(x)}{\nu_{1} G_{m}^{\alpha_{1}}(1)} \sum_{j=1}^{m} \frac{\beta_{j}}{\beta_{1}} G_{m}^{\alpha_{1}-\alpha_{j}}(0)=0
\end{gathered}
$$

и

$$
\begin{gathered}
{\left[\sum_{j=1}^{m} \frac{\beta_{j}}{\beta_{1}}\left(H(x-t) G_{m}^{\alpha_{1}-\alpha_{j}+1}(x-t)-G_{m}^{\alpha_{1}-\alpha_{j}}(1-t) \frac{1+\nu_{1} G_{m}^{\alpha_{1}+1}(x)}{\nu_{1} G_{m}^{\alpha_{1}}(1)}\right)\right]_{t=0}} \\
=\sum_{j=1}^{m} \frac{\beta_{j}}{\beta_{1}} G_{m}^{\alpha_{1}-\alpha_{j}+1}(x)-\frac{1+\nu_{1} G_{m}^{\alpha_{1}+1}(x)}{\nu_{1} G_{m}^{\alpha_{1}}(1)} \sum_{j=1}^{m} \frac{\beta_{j}}{\beta_{1}} G_{m}^{\alpha_{1}-\alpha_{j}}(1)=0 .
\end{gathered}
$$

Функцию $\mathscr{G}(x, t)$ назовем функцией Грина задачи (1)-(3).

Теперь покажем, что решение задачи (1)-(3) будет иметь вид (14). Заменив в уравнении (1) $x$ на $t$ и умножив обе части уравнения на функцию $\mathscr{G}=\mathscr{G}(x, t)$, проинтегрируем от 0 до 1 по переменной $t$, считая переменную $x$ фиксированной,

$$
\int_{0}^{1} \mathscr{G}(x, t) \sum_{j=1}^{m} \beta_{j} \partial_{0 t}^{\alpha_{j}} u(t) d t+\lambda \int_{0}^{1} \mathscr{G}(x, t) u(t) d t=\int_{0}^{1} \mathscr{G}(x, t) f(t) d t .
$$

Воспользовавшись соотношением (2) и формулой дробного интегрирования по частям $[17$, c. 15$]$

$$
\int_{0}^{1} g(s) D_{0 s}^{\gamma} h(s) d s=\int_{0}^{1} h(s) D_{1 s}^{\gamma} g(s) d s \quad(\gamma \leqslant 0),
$$

получаем

$$
\int_{0}^{1} u^{\prime \prime}(t) \sum_{j=1}^{m} \beta_{j} D_{1 t}^{\alpha_{j}-2} \mathscr{G}(x, t) d t+\lambda \int_{0}^{1} \mathscr{G}(x, t) u(t) d t=\int_{0}^{1} \mathscr{G}(x, t) f(t) d t .
$$


Проинтегрируем по частям первое слагаемое равенства (16):

$$
\int_{0}^{1} u^{\prime \prime}(t) \sum_{j=1}^{m} \beta_{j} D_{1 t}^{\alpha_{j}-2} \mathscr{G}(x, t) d t=\left.u^{\prime}(t) \sum_{j=1}^{m} \beta_{j} D_{1 t}^{\alpha_{j}-2} \mathscr{G}(x, t)\right|_{0} ^{1}-\int_{0}^{1} u^{\prime}(t) \sum_{j=1}^{m} \beta_{j} D_{1 t}^{\alpha_{j}-1} \mathscr{G}(x, t) d t .
$$

Разбив промежуток интегрирования на две части и учитывая свойства 2 и 4 , из последнего выражения имеем:

$$
\begin{aligned}
\left.u^{\prime}(t) \sum_{j=1}^{m} \beta_{j} D_{1 t}^{\alpha_{j}-2} \mathscr{G}(x, t)\right|_{0} ^{1}-\left(\int_{0}^{x} u^{\prime}(t) \sum_{j=1}^{m} \beta_{j} D_{1 t}^{\alpha_{j}-1} \mathscr{G}(x, t) d t\right. \\
\left.+\int_{x}^{1} u^{\prime}(t) \sum_{j=1}^{m} \beta_{j} D_{1 t}^{\alpha_{j}-1} \mathscr{G}(x, t) d t\right)=\left[u^{\prime}(t) \sum_{j=1}^{m} \beta_{j} D_{1 t}^{\alpha_{j}-2} \mathscr{G}(x, t)\right]_{t=1} \\
-\left[u^{\prime}(t) \sum_{j=1}^{m} \beta_{j} D_{1 t}^{\alpha_{j}-2} \mathscr{G}(x, t)\right]_{t=0}+u(x)+\int_{0}^{1} u(t) \sum_{j=1}^{m} \beta_{j} D_{1 t}^{\alpha_{j}} \mathscr{G}(x, t) d t .
\end{aligned}
$$

Полученное равенство подставляем в равенство (16)

$$
\begin{gathered}
{\left[u^{\prime}(t) \sum_{j=1}^{m} \beta_{j} D_{1 t}^{\alpha_{j}-2} \mathscr{G}(x, t)\right]_{t=1}-\left[u^{\prime}(t) \sum_{j=1}^{m} \beta_{j} D_{1 t}^{\alpha_{j}-2} \mathscr{G}(x, t)\right]_{t=0}} \\
+u(x)+\int_{0}^{1} u(t) \sum_{j=1}^{m} \beta_{j} D_{1 t}^{\alpha_{j}} \mathscr{G}(x, t) d t+\lambda \int_{0}^{1} \mathscr{G}(x, t) u(t) d t=\int_{0}^{1} \mathscr{G}(x, t) f(t) d t,
\end{gathered}
$$

откуда, учитывая (15), приходим к формуле (14). Таким образом, доказано, что если задача (1)-(3) имеет решение, то оно представимо в виде (14). Отсюда в частности следует единственность решения при выполнении условия (13).

Теперь покажем, что функция (14) является решением задачи (1)-(3). Формулу (14) можно переписать в виде

$$
u(x)=a u_{1}(x)-b u_{2}(x)-u_{3}(x)+u_{4}(x)
$$

где

$$
\begin{gathered}
u_{1}(x)=\left[\sum_{j=1}^{m} \beta_{j} D_{1 t}^{\alpha_{j}-2} \mathscr{G}(x, t)\right]_{t=0}, \quad u_{2}(x)=\left[\sum_{j=1}^{m} \beta_{j} D_{1 t}^{\alpha_{j}-2} \mathscr{G}(x, t)\right]_{t=1}, \\
u_{3}(x)=\frac{1+\nu_{1} G_{m}^{\alpha_{1}+1}(x)}{\beta_{1} \nu_{1} G_{m}^{\alpha_{1}}(1)} \int_{0}^{1} f(t) G_{m}^{\alpha_{1}-1}(1-t) d t, \quad u_{4}(x)=\frac{1}{\beta_{1}} \int_{0}^{x} f(t) G_{m}^{\alpha_{1}}(x-t) d t .
\end{gathered}
$$

Учитывая равенства (9)-(12), получаем

$$
\begin{gathered}
u_{1}(x)=x+\nu_{1} G_{m}^{\alpha_{1}+2}(x)-\frac{\left(1+\nu_{1} G_{m}^{\alpha_{1}+1}(x)\right)\left(1+\nu_{1} G_{m}^{\alpha_{1}+1}(1)\right)}{\nu_{1} G_{m}^{\alpha_{1}}(1)}, \\
u_{2}(x)=\frac{1+\nu_{1} G_{m}^{\alpha_{1}+1}(x)}{\nu_{1} G_{m}^{\alpha_{1}}(1)} .
\end{gathered}
$$


Из (12) следует, что каждая из функций $u_{1}(x), u_{2}(x), u_{3}(x)$ удовлетворяют однородному уравнению

$$
\left(\sum_{j=1}^{m} \beta_{j} \partial_{0 x}^{\alpha_{j}}+\lambda\right) u_{i}(x)=0, \quad i=1,2,3 .
$$

Соотношения (9), (10) и (11) показывают выполнение краевых условий (3).

Для завершения доказательства остается показать, что

$$
\left(\sum_{j=1}^{m} \beta_{j} \partial_{0 x}^{\alpha_{j}}+\lambda\right) u_{4}(x)=f(x) .
$$

Имея ввиду (9) и

$$
\sum_{i=2}^{m} \nu_{i} G_{m}^{\gamma_{i}+\mu}(x)=G_{m}^{\mu}(x)-\nu_{1} G_{m}^{\alpha_{1}+\mu}(x)-\frac{x^{\mu-1}}{\Gamma(\mu)},
$$

(см. (10) и (11)), а также закон композиции операторов дробного интегро-дифференцирования [1, с. 87], получаем

$$
\begin{gathered}
\sum_{i=1}^{m} \frac{\beta_{i}}{\beta_{1}} \partial_{0 x}^{\alpha_{i}} \int_{0}^{x} f(t) G_{m}^{\alpha_{1}}(x-t) d t=\frac{d}{d x} \sum_{i=1}^{m} \frac{\beta_{i}}{\beta_{1}} D_{0 x}^{\alpha_{i}-\alpha_{1}}\left(\int_{0}^{x} D_{0 x}^{\alpha_{1}-2} g(t) G_{m}^{1}(x-t) d t\right) \\
=\frac{d}{d x} \sum_{i=1}^{m} \frac{\beta_{i}}{\beta_{1}} D_{0 x}^{\alpha_{i}-\alpha_{1}} \int_{0}^{x} f(t) G_{m}^{1}(x-t) d t=\frac{d}{d x} \int_{0}^{x} f(t)\left(\nu_{1} G_{m}^{\alpha_{1}+1}(x-t)+1\right) d t \\
=\frac{-\lambda}{\beta_{1}} \int_{0}^{x} f(t) G_{m}^{\alpha_{1}}(x-t) d t+f(x),
\end{gathered}
$$

откуда следует (17). $\triangleright$

ЗАмечАниЕ. При положительных значениях параметров $a, b$ и аргумента $z$ функция Райта $\phi(a, b ; z)$ принимает положительные значения. Следовательно, при

$$
\lambda<0, \quad \beta_{j} \leqslant 0, \quad \beta_{1}>0
$$

функция, определяемая равенством (5), тоже является положительной. Тогда функция $G_{m}^{\mu}(x)>0$ для любого $\mu>0$. Таким образом, условия (18) обеспечивают выполнение (13) для всех $m \in \mathbb{N}$ и $\left.\alpha_{j} \in\right] 1, \alpha_{1}\left[, \alpha_{1} \in\right] 1,2[$.

3. Интегральное представление функции $G_{m}^{\mu}(x)$. Обозначим через

$$
\gamma(r, \omega \pi)=\{z:|z|=r,|\arg z| \leqslant \omega \pi\} \cup\{z:|z| \geqslant r, \arg z= \pm \omega \pi\},
$$

$1 / 2<\omega \leqslant 1-$ контур Ханкеля, направление обхода выбрано в сторону неубывания $\arg z$ $[17$, c. 12$]$.

Лемма. Для функции $G_{m}^{\mu}(x)$, при $\lambda>0$, с параметрами (6) и (8) справедливо следующее интегральное представление:

$$
G_{m}^{\mu}(x)=\frac{\beta_{1}}{2 \pi i} \int_{\gamma(r, \omega \pi)} e^{x p} \frac{p^{\alpha_{1}-\mu}}{\sum_{j=1}^{m} \beta_{j} p^{\alpha_{j}}+\lambda} d p .
$$


$\triangleleft$ Используя интегральное представление функции Райта [17, с. 23], получаем

$$
x^{\mu_{j}-1} \phi\left(\gamma_{j}, \mu_{j} ; z_{j} x^{\gamma_{j}}\right)=\frac{1}{2 \pi i} \int_{\gamma(r, \omega \pi)} e^{x p} p^{-\mu_{j}} e^{z_{j} p^{-\gamma_{j}}} d p .
$$

Подставив последнее равенство в (6) и пользуясь теоремой умножения [18, с. 63], имеем

$$
\begin{gathered}
S_{m}^{\mu}\left(x ; \nu_{1} t, \ldots, \nu_{m} t ; \gamma_{1}, \ldots, \gamma_{m}\right)=\left(h_{1} * h_{2} * \cdots * h_{m}\right)(x) \\
=\frac{1}{2 \pi i} \int_{\gamma(r, \omega \pi)} e^{x p} p^{-\mu} \exp \left(t \sum_{j=1}^{m} \nu_{j} p^{-\gamma_{j}}\right) d p .
\end{gathered}
$$

Домножим (21) на $e^{-t}$ и проинтегрируем по $t$ от 0 до $\infty$. В (19) параметр $r$ можно выбрать так, чтобы $\operatorname{Re}\left(1-\left(\sum_{j=1}^{m} \nu_{j} p^{\gamma_{j}}\right)>0\right.$. Поэтому в равенстве $(21)$ возможно изменение порядка интегрирования, с учетом (6) отсюда следует

$$
\begin{aligned}
G_{m}^{\mu}(x)=\frac{1}{2 \pi i} \int_{\gamma(r, \omega \pi)} e^{x p} p^{-\mu} \int_{0}^{\infty} \exp (-t+ & \left.t \sum_{j=1}^{m} \nu_{j} p^{-\gamma_{j}}\right) d t d p \\
& =\frac{\beta_{1}}{2 \pi i} \int_{\gamma(r, \omega \pi)} e^{x p} \frac{p^{\alpha_{1}-\mu}}{\beta_{1} p^{\alpha_{1}}+\sum_{j=2}^{m} \beta_{j} p^{\alpha_{j}}+\lambda} d p . \triangleright
\end{aligned}
$$

4. О вещественных собственных значениях. Выражение $\nu_{1} G_{m}^{\alpha_{1}}(1)$ будем рассматривать как функцию параметра $\lambda$. Обозначим $\psi(\lambda)=\nu_{1} G_{m}^{\alpha_{1}}(1)$. Тогда условие (13) примет вид $\psi(\lambda) \neq 0$. Из (20) следует, что

$$
G_{m}^{\mu}(1)=\frac{\beta_{1}}{2 \pi i} \int_{\gamma(r, \omega \pi)} \frac{e^{p}}{\sum_{j=1}^{m} \beta_{j} p^{\alpha_{j}}+\lambda} d p
$$

Теорема 2. Пусть $\lambda>0$ и

$$
\sum_{j=1}^{m} \frac{\beta_{j}}{\Gamma\left(-\alpha_{j}\right)} \neq 0
$$

Тогда задача Неймана (3) для уравнения (1) имеет единственное решение для всех (за исключением, быть может, конечного числа) значений $\lambda$.

$\triangleleft$ Для доказательства теоремы необходимо показать, что соотношение (13) выполняется для всех $\lambda \in \mathbb{R}$, за исключением не более чем конечного числа его значений. 
Рассмотрим следующее предельное соотношение:

$$
\begin{aligned}
& \lim _{\lambda \rightarrow \infty} \lambda \psi(\lambda)=-\lim _{\lambda \rightarrow \infty} \frac{\lambda^{2}}{2 \pi i} \int_{\gamma(r, \omega \pi)} \frac{e^{p}}{\sum_{j=1}^{m} \beta_{j} p^{\alpha_{j}}+\lambda} d p \\
& =-\lim _{\lambda \rightarrow \infty} \frac{\lambda}{2 \pi i} \int_{\gamma(r, \omega \pi)} e^{p} \frac{\lambda-\sum_{j=1}^{m} \beta_{j} p^{\alpha_{j}}+\sum_{j=1}^{m} \beta_{j} p^{\alpha_{j}}}{\sum_{j=1}^{m} \beta_{j} p^{\alpha_{j}}+\lambda} d p \\
& =-\lim _{\lambda \rightarrow \infty} \frac{\lambda}{2 \pi i} \int_{\gamma(r, \omega \pi)} e^{p}\left(1-\frac{\sum_{j=1}^{m} \beta_{j} p^{\alpha_{j}}}{\sum_{j=1}^{m} \beta_{j} p^{\alpha_{j}}+\lambda}\right) d p .
\end{aligned}
$$

Так как

$$
\frac{1}{2 \pi i} \int_{\gamma(r, \omega \pi)} e^{p} d p=0,
$$

то согласно [19, с. 311] получим

$$
\begin{gathered}
\lim _{\lambda \rightarrow \infty} \lambda \psi(\lambda)=\lim _{\lambda \rightarrow \infty} \frac{1}{2 \pi i} \int_{\gamma(r, \omega \pi)} e^{p} \frac{\sum_{j=1}^{m} \beta_{j} p^{\alpha_{j}}}{\lambda^{-1} \sum_{j=1}^{m} \beta_{j} p^{\alpha_{j}}+1} d p \\
=\lim _{\lambda \rightarrow \infty} \frac{1}{2 \pi i} \int_{\gamma(r, \omega \pi)} e^{p} \sum_{j=1}^{m} \beta_{j} p^{\alpha_{j}} d p=\sum_{j=1}^{m} \frac{\beta_{j}}{\Gamma\left(-\alpha_{j}\right)} .
\end{gathered}
$$

Из $(20)$ следует, что $\psi(\lambda)$ является целой функцией параметра $\lambda$. В силу того, что $\psi(\lambda)$ является целой, в ограниченной области она может иметь лишь конечное число вещественных нулей. Поэтому, если предположить, что функция $\psi(\lambda)$ имеет бесконечное число нулей, то они должны сгущаться в бесконечно удаленной точке, что противоречит (22) и (23). Следовательно, условие (13) может нарушаться только для конечного числа значений $\lambda$.

\section{Литература}

1. Нахушев А. М. Дробное исчисление и его применение.-М.: Физматлит, 2003.-272 с.

2. Barrett J. H. Differential equations of non-integer order // Canadian J. Math.-1954.-Vol. 6, № 4.P. 529-541.

3. Джрбашян М. М., Нерсесян А. Б. Дробные производные и задача Коши для дифференциальных уравнений дробного порядка // Изв. АН Армянской ССР. Математика.-1968.-Т. 3, № 1.-С. 3-28.

4. Джрбашян M. M. Краевая задача для дифференциального оператора дробного порядка типа Штурма - Лиувилля // Изв. АН Армянской ССР.-1970.-№ 2.-С. 71-96.

5. Нахушев А. М. Задача Штурма - Лиувилля для обыкновенного дифференциального уравнения второго порядка с дробными производными в младших членах // Докл. АН CCCP.-1977.-T. 234, № 2.-C. $308-311$.

6. Ozturk I. On the theory of fractional differential equation // Докл. Адыгской (Черкесской) Международной академии наук.-1998.-Т. 3, № 2.-С. 35-39. 
7. Псху А. В. К теории задачи Коши для линейного обыкновенного дифференциального уравнения дробного порядка // Докл. Адыгской (Черкесской) междунар. акад. наук.-2009.-Т. 11, № 1.C. $61-65$.

8. Псху А. В. Начальная задача для линейного обыкновенного дифференциального уравнения дробного порядка // Мат. сб.-2011.-Т. 202, № 4.-С. 111-122.

9. Гадзова Л. Х. Обобщенная задача Дирихле для линейного дифференциального уравнения дробного порядка с постоянными коэффициентами // Диф. уравнения.-2014.-Т. 50, № 1.-С. 121-125.

10. Kilbas A. A., Srivastava H. M., Trujillo J. J. Theory and Applications of Fractional Differential Equations.-Amsterdam: Elsevier, 2006.-(North-Holland Math. Stud.; Vol. 204).

11. Гадзова Л. Х. Задача Дирихле для обыкновенного дифференциального уравнения дробного порядка // Докл. Адыгской (Черкесской) междунар. акад. наук.-2013.-Т. 15, № 2.-С. 36-39.

12. Гадзова Л. X. K теории краевых задач для дифференциального уравнения дробного порядка с производной Капуто // Докл. Адыгской (Черкесской) междунар. акад. наук.-2014.-Т. 16, № 2.C. $34-40$.

13. Bagley R. L., Torvik P. J. Fractional calculus in the transient analysis of viscoelastically damped structures // AIAA J.-1985.-Vol. 23, № 6.-P. 918-925.

14. Самко С. Г., Килбас А. А., Маричев О. И. Интегралы и производные дробного порядка и некоторые их приложения.-Минск.: Наука и техника, 1987.-688 с.

15. Нахушев А. М., Тхакахов Р. Б. О континуальных аналогах реологических уравнений состояния и логистическом законе изменения вязкоупругих свойств полимера // Докл. Адыгской (Черкесской) междунар. акад. наук.-1995.-Т. 1, № 2.-С. 6-11.

16. Wright E. M. On the coefficients of power series having exponential singularities // J. London Math. Soc.-1933.-Vol. 8, № 29.-P. 71-79.

17. Псху А. В. Уравнения в частных производных дробного порядка.-М.: Наука, 2005.-199 с.

18. Штокала И. 3. Операционное исчисление (обобщение и приложения).-Киев: Наукова думка, 1972.-304 c.

19. Кудрявцев Л. Д. Курс математического анализа. Т. 2.-М.: Высшая школа, 1981.-584 с.

Статвя поступила 1 апреля 2015 г.

ГАДЗОВА ЛуИЗА ХАмидБИЕВНА

Институт прикладной математики и автоматизации,

научный сотрудник отдела дробного исчисления

РОССИЯ, 360000, г. Нальчик, ул. Шортанова, 89 а

E-mail: macaneeva@mail.ru

\section{NEUMANN PROBLEM FOR AN ORDINARY DIFFERENTIAL EQUATION OF FRACTIONAL ORDER}

\section{Gadzova L. H.}

A linear ordinary differential equation of fractional order with constant coefficients is considered in the paper. Such equation should be subsumed into the class of discretely distributed order, or multi-term differential equations. The fractional differentiation is given by the Caputo derivative. We solve The Nuemann problem for the equation under study, prove the existence and uniqueness of the solution, find an explicit representation for solution in terms of the Wright function, and construct the respective Green function. It is also proves that the real part of the spectrum of the problem may consist at most of a finite number of eigenvalues.

Key words: boundary value problem, operator of fractional differentiation, Riemann-Liouville operator, Caputo operator. 